\title{
Anisotropy in Cosmic-Ray Arrival Directions Using IceCube and IceTop
}

\author{
The IceCube Collaboration \\ ${ }^{\dagger}$ http://icecube.wisc.edu/collaboration/authors/icrc15_icecube \\ E-mail: westerhoffephysics.wisc.edu
}

The IceCube Neutrino Observatory recorded more than 250 billion cosmic-ray induced muon events between May 2009 and May 2014. We use this data set to study the anisotropy in the arrival direction distribution of cosmic rays in the $\mathrm{TeV}$ to $\mathrm{PeV}$ energy range. The anisotropy features large regions of relative excess and deficit with amplitude on the order of $10^{-3}$. A decomposition of the arrival direction distribution into spherical harmonics shows that most of the power is contained in the low-multipole $(\ell \leq 4)$ moments. However, higher multipole components are also statistically significant down to an angular scale of less than $10^{\circ}$. The data set also allows for a detailed study of the anisotropy for various cosmic-ray median energies. The large-scale structure observed at energies near $20 \mathrm{TeV}$ reaches a minimum amplitude, accompanied by a change in phase, around $150 \mathrm{TeV}$. At higher energies, we observe a strong deficit with an amplitude increasing with energy up to $5 \mathrm{PeV}$, the highest energies currently accessible to IceCube. The deficit is also present in IceTop maps of similar energies. No time-dependence of the large-scale structure was observed in the five-year period covered by this analysis.

Corresponding authors: F.T. McNally ${ }^{1}$, P. Desiati ${ }^{1}$, and S. Westerhoff*1

${ }^{1}$ Wisconsin IceCube Particle Astrophysics Center (WIPAC) and Department of Physics, University of Wisconsin-Madison, Madison, WI 53706, USA

The 34th International Cosmic Ray Conference,

30 July-6 August, 2015

The Hague, The Netherlands

\footnotetext{
* Speaker.
} 


\section{Introduction}

Galactic magnetic fields sufficiently scramble the arrival directions of cosmic rays with $\mathrm{TeV}$ energies to prohibit any direct identification of sources based on their arrival direction distribution. However, cosmic rays at this energy propagate diffusively through the Galaxy, and their distribution in the sky shows a degree of anisotropy at the per-mille level that might give some indications of the location of nearby sources and the process of propagating from their sources to us.

Measurements of anisotropy in the arrival direction distribution of $\mathrm{TeV}$ cosmic rays have been published by a number of experiments, including the Tibet AS $\gamma$ [1], Super-Kamiokande [2, 3], Milagro [4, 5], EAS-TOP [6], MINOS [7], ARGO-YBJ [8], and HAWC [9] experiments in the Northern Hemisphere and IceCube $[10,11,12]$ and its surface air shower array IceTop [13] in the Southern Hemisphere.

In both hemispheres, the observed anisotropy has two main features: a large-scale structure with an amplitude of about $10^{-3}$ usually fitted as a dipole or a sum of low-order multipoles, and a small-scale structure with a few localized regions of cosmic-ray excesses and deficits of angular size $10^{\circ}$ to $30^{\circ}$. The large-scale structure is usually interpreted as a result of diffusive propagation, with the maximum of the dipole possibly pointing in the direction of one or several nearly sources (see for example [14]). On the other hand, the misalignment between the cosmic-ray density gradient and the regular Galactic magnetic field would prevent pointing to any specific source, although it would suppress the anisotropy amplitude to a value closer to what is observed [15]. The smallscale structure is more difficult to explain. It could be the product of turbulence in the Galactic magnetic field [16, 17], among other explanations.

The IceCube detector at the geographic South Pole is currently the only detector that can study cosmic-ray anisotropy in the Southern Hemisphere. Based on data taken with partial detector configurations while the detector was still under construction, we have previously published observations of cosmic-ray anisotropy with IceCube [10, 11] and IceTop [13], as well as studies of the energy dependence of the anisotropy [12]. In this paper, we update the previous results using all of the currently available data, collected in 1690 days of livetime between May 2009 and May 2014. During this period, IceCube recorded about 250 billion events and IceTop recorded 170 million events.

\section{The Data Set}

IceCube is a cubic-kilometer neutrino detector installed in the ice at the geographic South Pole [18]. High-energy neutrinos are detected by observing the Cherenkov radiation from charged particles produced by neutrino interactions in the ice or in the bedrock below the detector. The Cherenkov light is detected by an array of 5160 digital optical modules (DOMs) deployed at depths between $1450 \mathrm{~m}$ and $2450 \mathrm{~m}$ below the surface of the ice sheet. Each DOM is a pressure-resistant glass sphere that contains a 10-inch photomultiplier tube (PMT) and electronics to digitize the signals. The DOMs are attached to 86 vertical strings separated by an average distance of $125 \mathrm{~m}$, each string hosting 60 DOMs equally spaced over the kilometer of instrumented length.

The IceTop air-shower array is located on the surface of the ice sheet above the IceCube neutrino detector [19]. An integral part of IceCube, IceTop is a dedicated cosmic-ray detector 
optimized for air-shower observations at $\mathrm{PeV}$ energies. It consists of 81 surface stations, with two light-tight tanks per station. Each tank measures $1.8 \mathrm{~m}$ in diameter, is filled with highly transparent ice of $0.9 \mathrm{~m}$ in height, and contains two DOMs with different gains.

Both the neutrino detector buried deep in the ice and the cosmic-ray shower detector on the surface can be used to study cosmic-ray arrival direction anisotropy. The in-ice component detects cosmic rays through the relativistic muons produced by cosmic-ray air showers. The trigger rate for events with eight or more DOMs in coincidence (see [20] for details) varies between $2 \mathrm{kHz}$ and $2.4 \mathrm{kHz}$, with the modulation caused by seasonal variations of the stratospheric temperature [21]. The detected muon events are generated by primary cosmic-ray particles with median energy of $20 \mathrm{TeV}$, as determined by simulations. The air-shower muons preserve the arrival direction of the primary cosmic ray to within about $0.2^{\circ}$. Because of the high trigger rate and the limited data transfer bandwidth available from the South Pole, all cosmic-ray data are stored in a compact data storage and transfer (DST) format, containing only the results of a fast angular reconstruction and some limited information per event. The median angular resolution for this data set, as determined by simulation, is $3^{\circ}$. The DST data format does not contain event-by-event errors on the arrival direction reconstruction.

The IceTop air-shower array detects cosmic rays at a rate of approximately $30 \mathrm{~Hz}$ with a minimum primary particle energy threshold of about $400 \mathrm{TeV}$. IceTop is sensitive to the electromagnetic component of the shower, not just the muonic component. Due to transmission bandwidth limitations, IceTop data is subject to prescaling. The prescaling factor depends on the event size and has changed over time with the detector configuration. Only events that trigger eight or more stations have not been prescaled for any configuration, so to obtain a consistent data set for the entire period covered by this analysis, we used only this subset of showers. The resulting data set has a median energy of $1.7 \mathrm{PeV}$.

In the first two years used in this analysis, IceCube and IceTop operated in partial detector configurations, with 59 active strings/stations (IC59/IT59) from May 2009 to May 2010, and 79/73 strings/stations (IC79/IT73) from May 2010 to May 2011. Since May 2011, the detector has been operating in its full configuration (IC86/IT81).

\section{Large- and Small-Scale Structure}

Several steps are necessary to produce sky maps of the large- and small-scale cosmic-ray anisotropy. For our analysis, we implemented an equal-area binning of the sky using the HEALPix library [22]. The underlying resolution is about $1^{\circ}$, but the final maps are smoothed on angular scales corresponding to the resolution of the detector.

The first step in the study of anisotropy in cosmic-ray arrival directions is the creation of a reference map showing the response of the detector to an isotropic flux of cosmic rays. The reference map itself is not isotropic, as it accounts for changes in the cosmic-ray rate from atmospheric effects, occasional detector downtime, and effects from the detector geometry. The reference map is generated from the data themselves. For each event in the data map, events in the reference map are created by attaching the same arrival direction in local coordinates to randomly selected arrival times from the distribution of real arrival times within a time window of 24 hours. The technique is described in detail in [11]. We note that this technique only modifies the right ascension of the 

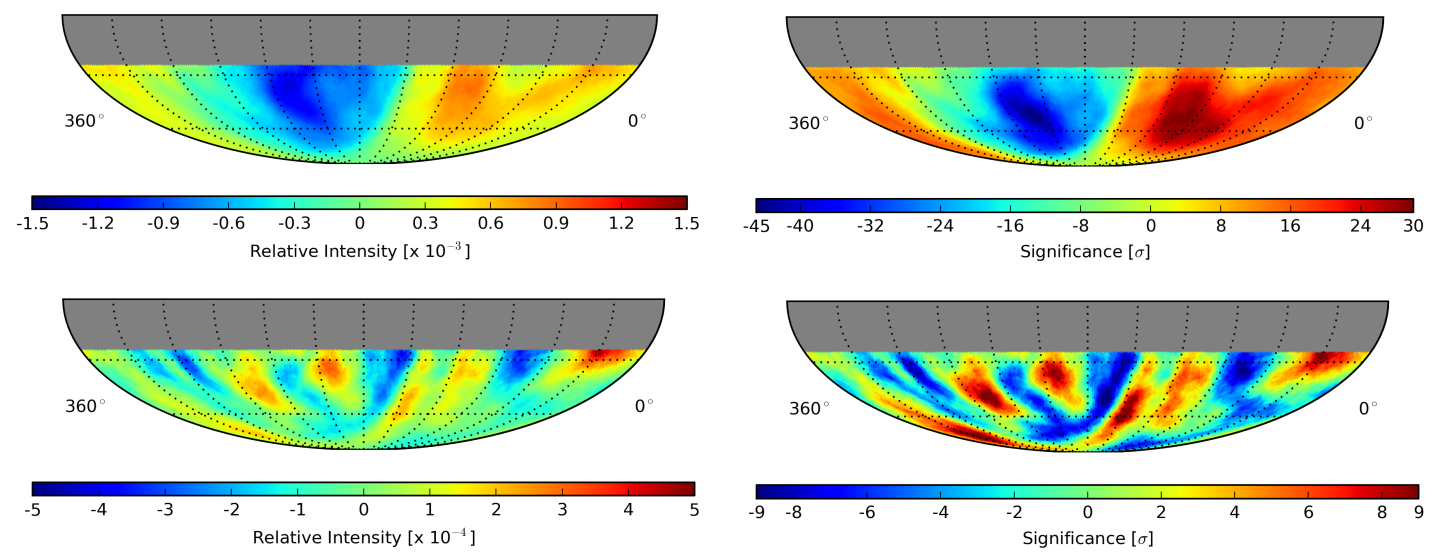

Figure 1: Equatorial maps of the relative intensity of the cosmic-ray flux (left) and the pre-trial statistical significance of the deviation from isotropy (right) before (top) and after (bottom) dipole and quadrupole subtraction. An angular smoothing with $5^{\circ}$ radius is applied to all maps.

event within the same declination band. Consequently, any large-scale structure in the arrival direction distribution is reduced to its projection onto the equatorial plane. However, simulations show that localized (small-scale) structures are correctly reproduced with this method.

In the second step, the reference map is compared to the data map to obtain a sky map that shows the deviations from isotropy. Typically, these maps show the relative intensity of the cosmicray flux, $\delta I_{i}=\left(N_{i}-\langle N\rangle_{i}\right) /\langle N\rangle_{i}$, where $N_{i}$ and $\langle N\rangle_{i}$ are the number of observed events and the number of reference events in the $i^{\text {th }}$ bin of the map, respectively. To evaluate the statistical significance of any deviation from anisotropy, we also produce maps of significance calculated according to $\mathrm{Li}$ \& Ma [23]. A top-hat smoothing procedure in which a single pixel's value is the sum of all pixels within a $5^{\circ}$ radius has been applied to all maps.

Figure 1 (top) shows, in equatorial coordinates, the sky map of relative intensity (left) and statistical significance (right) for five years of IceCube data. No energy cuts have been applied for these maps, so the median energy of the cosmic-ray primaries is $20 \mathrm{TeV}$. The maps are dominated by large-scale structure, with a deep deficit from $150^{\circ}$ to $250^{\circ}$ in right ascension and a corresponding excess from $30^{\circ}$ to $120^{\circ}$. The distribution is, however, not well described by a dipole or even a sum of the first low-order multipole moments (dipole, quadrupole, ...) of the spherical harmonic function, indicating the presence of structure at smaller angular scales. To study this small-scale anisotropy, the dominating large-scale structure has to be removed. To achieve this, the dipole and quadrupole terms are fit to the relative intensity map and then subtracted.

The bottom panels of Figure 1 show maps of relative intensity (left) and statistical significance (right) after the subtraction of the best-fit dipole and quadrupole terms. The regions of excess and deficit in cosmic-ray flux are similar to those shown in previous work [11], but the increased statistics of the five-year data set increases the significance of the features and leads to a better resolution of the small-scale structure. The maps clearly indicate statistically significant anisotropy down to angular scales approaching the angular resolution of the detector.

Figure 2 shows the angular power spectrum of the five-year data set both before (blue) and after (red) the subtraction of the dipole and quadrupole moments. It confirms the presence of significant 


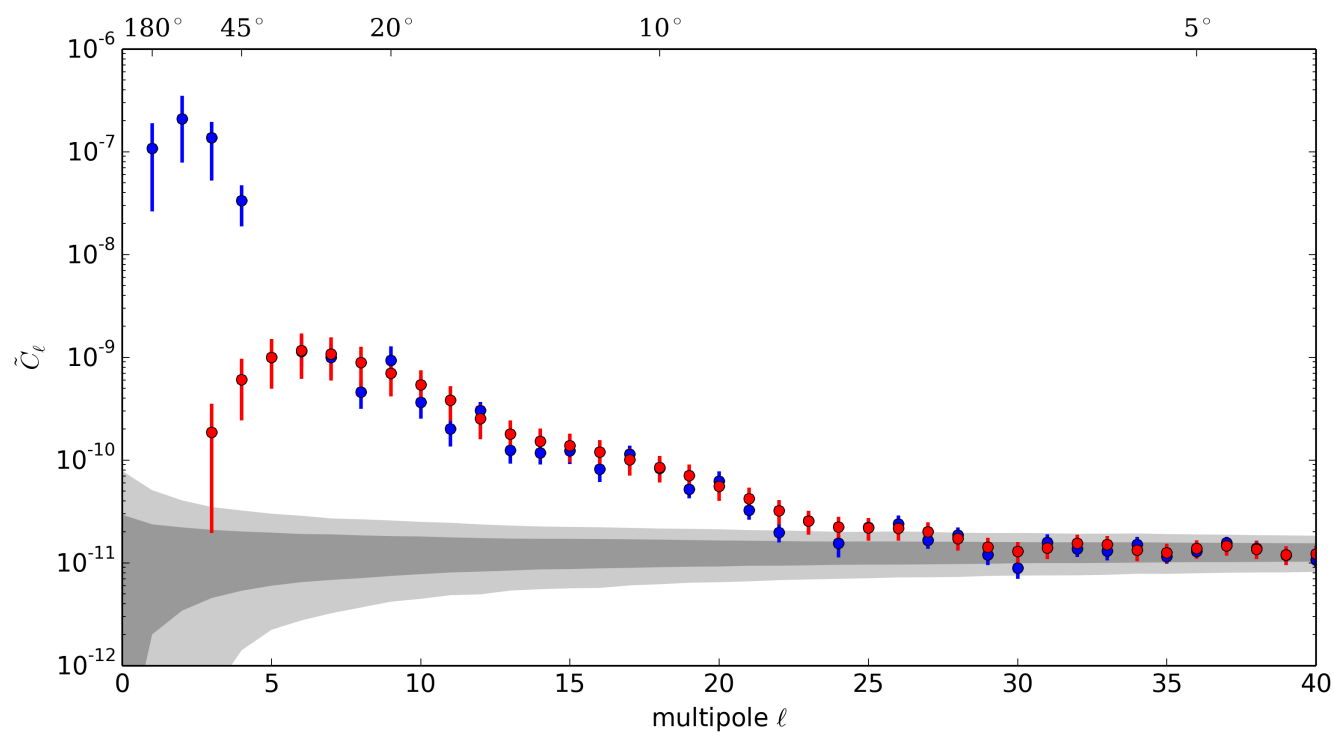

Figure 2: Angular power spectrum for five years of IceCube data (blue) and for the same date with best-fit dipole and quadrupole moments subtracted $(r e d)$. The amplitudes $C_{\ell}$ for $\ell=1,2$ are consistent with 0 after the subtraction. Dark- and light-gray bands represent the power spectra for isotropic sky maps at the $68 \%$ and $90 \%$ confidence levels, respectively.

structure up to multipoles $\ell \simeq 20$, corresponding to angular scales of less than $10^{\circ}$.

\section{Energy Dependence}

Studies of the arrival direction anisotropy between $\mathrm{TeV}$ and $\mathrm{PeV}$ energies have revealed a substantial change in the amplitude and phase of the best-fit dipole with energy [6, 12]. To analyze the energy dependence, we split the data set into nine bins with increasing median energy based on the number of DOMs that collected Cherenkov light and the reconstructed zenith angle (see [12] for details). The energy resolution is poor (on the order of $0.5 \log (E / \mathrm{GeV})$ ) because muons transport to the surface a small fraction of the total shower energy, with relatively large fluctuations. Therefore, events in each bin have a wide energy distribution and the distributions of the different energy bins overlap substantially. However, the data in the nine energy bins are statistically independent.

Figure 3 shows the dipole amplitude (left) and phase (right) as a function of energy. To obtain these values, we first produce a projection of the relative intensity onto right ascension and then perform a one-dimensional fit of the full set of harmonic functions to the projection. We fit this projection rather than the full sky map because the two-dimensional fit of spherical harmonics to the map is difficult to perform with a limited field of view. As a result of the method we applied to generate the reference map, the sky map will in any case only show the projection of any dipole component, so the one-dimensional fit is sufficient to study the energy dependence of the dominant dipole. The figure shows that a rapid shift of phase by almost $180^{\circ}$ occurs at an energy between $130 \mathrm{TeV}$ and $240 \mathrm{TeV}$. The dipole amplitude decreases with energy up to these energies, and increases again at higher energies. The red data point in Figure 3 is based on IceTop data with a median energy of $1.7 \mathrm{PeV}$. While the phase agrees well with that found in IceCube data at similar 

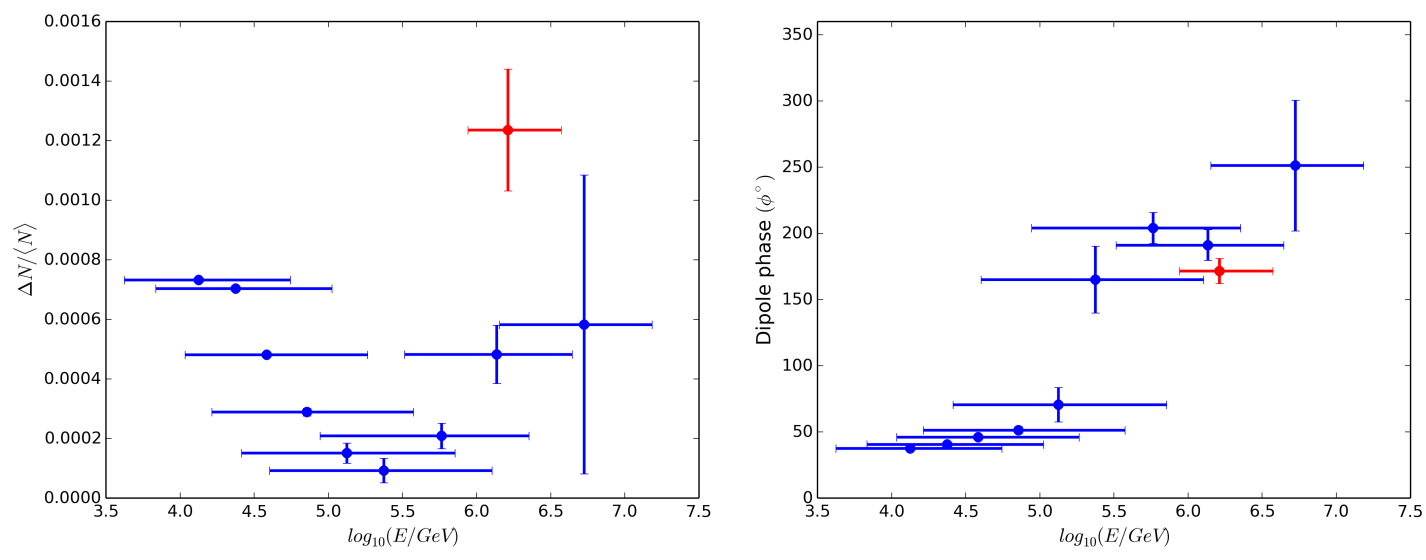

Figure 3: Amplitude (left) and $\phi$-component of phase (right) of dipole fit to IceCube (blue) and IceTop (red) sky maps for various energy bins. Data points indicate the median energy of each energy bin, with error bars showing the $68 \%$ containment interval.

energies, the amplitude of the anisotropy is larger in IceTop than in any IceCube energy bin. This could indicate a difference in the energy distribution and the chemical composition of IceCube and IceTop events and is currently under study.

\section{Time Dependence}

The data used in this analysis accumulated over a period of five years and therefore also allows for a study of the stability of the anisotropy over this time period. Such studies have been performed by other experiments, with contradictory results. Both the Tibet [24] and ARGO-YBJ experiments [8] have observed no significant variation in the anisotropy, whereas Milagro [5] reports a steady increase in the amplitude of the deficit region over a period of seven years (2000-2007). The discovery of time modulation in the shape of the anisotropy, in particular a variation with the 11year solar cycle, could be evidence for a heliospheric influence on the arrival directions.

Figure 4 shows the one-dimensional projection of the relative intensity onto right ascension for each year of IceCube data. Systematic errors are estimated by calculating the maximum amplitude of the signal in the anti-sidereal time frame (see [11] for details). A $\chi^{2}$-test shows that within errors, the large-scale anisotropy is stable over the five years of IceCube data. This is in agreement with the results of a study of the stability over a period of 12 years (2000-2012) using data recorded with the AMANDA and IceCube detector [25].

\section{Summary and Outlook}

The analysis of five years of data taken with the IceCube detector and its air-shower array IceTop show an energy-dependent anisotropy in the arrival direction distribution of $\mathrm{TeV}$ to $\mathrm{PeV}$ cosmic rays in the Southern Hemisphere. In addition to a large-scale structure, we observe significant small-scale structure down to scales approaching the angular resolution of the detector. The phase of the large-scale anisotropy changes rapidly between $130 \mathrm{TeV}$ and $240 \mathrm{TeV}$. During the time period analyzed, the large-scale structure is stable with time. 


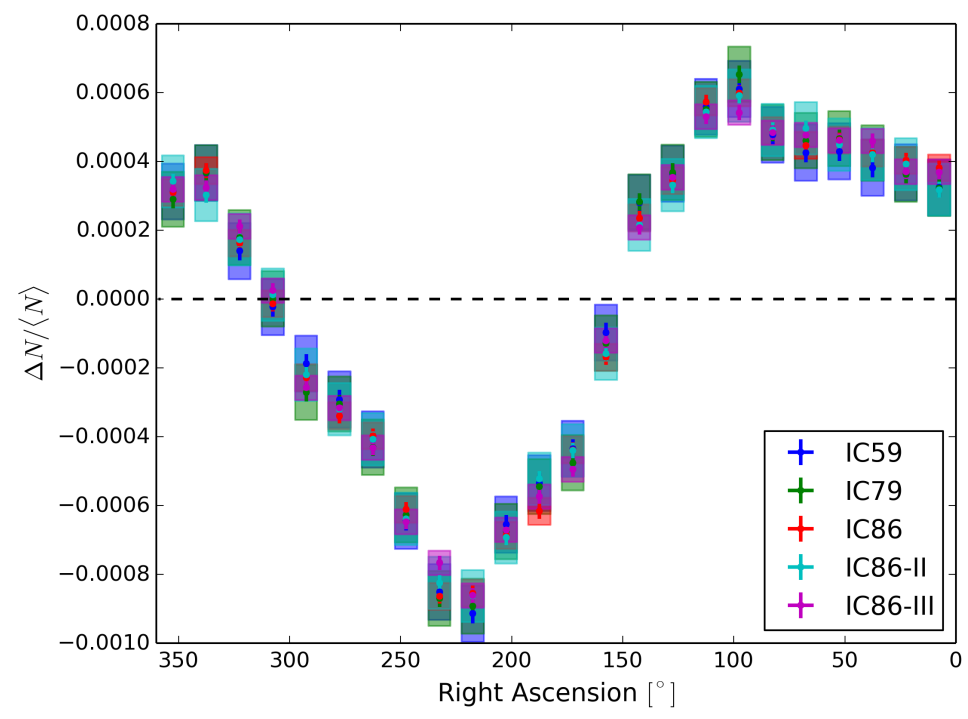

Figure 4: Relative intensity of the cosmic-ray flux as a function of right ascension for each configuration of the IceCube detector from IC59 to the third year of IC86. The plot is a one-dimensional projection of the sky map onto the right ascension axis. Systematic errors are calculated using the anti-sidereal frame for each year independently.

Due to the high data rate, the event-by-event information stored for the muon tracks in the in-ice detector is limited and does not allow for a more detailed analysis of the anisotropy. For data taken with the IceTop air-shower array, a more accurate energy reconstruction is available, and it is possible to study the energy spectrum of excess and deficit regions and a possible dependence of the anisotropy on the chemical composition of the primary cosmic-ray flux. Studies with detectors in the Northern Hemisphere have shown that the energy spectrum of the cosmic-ray flux in the most dominant excess regions is harder than the isotropic cosmic-ray flux $[4,8,9]$. In the near future, we plan to use IceTop data to search for similar effects in the southern sky.

\section{References}

[1] Tibet AS $\gamma$ Collaboration, M. Amenomori et al., Large-Scale Sidereal Anisotropy of Galactic Cosmic-Ray Intensity Observed by the Tibet Air Shower Array, Astrophys. J. 626 (2005) L29.

[2] Kamiokande Collaboration, K. Munakata et al., Large-Scale Anisotropy of the Cosmic-Ray Muon Flux in Kamiokande, Phys. Rev. D 56 (1997) 23.

[3] Super-Kamiokande Collaboration, G. Guillian et al., Observation of the Anisotropy of $10 \mathrm{TeV}$ Primary Cosmic Ray Nuclei Flux with the Super-Kamiokande-I Detector, Phys. Rev. D 75 (2007) 062003.

[4] Milagro Collaboration, A.A. Abdo et al., Discovery of Localized Regions of Excess 10-TeV Cosmic Rays, Phys. Rev. Lett. 101 (2008) 221101.

[5] Milagro Collaboration, A.A. Abdo et al., The Large-Scale Cosmic-Ray Anisotropy as Observed with Milagro, Astrophys. J. 69821212009.

[6] EAS-TOP Collaboration, M. Aglietta et al., Evolution of the Cosmic-Ray Anisotropy Above $10^{14} \mathrm{eV}$, Astrophys. J. 692 (2009) L130. 
[7] MINOS Collaboration, J.K. deJong et al., Observations of Large Scale Sidereal Anisotropy in 1 and $11 \mathrm{TeV}$ Cosmic Rays from the MINOS Experiment, Proc. 32nd ICRC, Beijing, China (2011) [arXiv:1201.2621].

[8] ARGO-YBJ Collaboration, B. Bartoli et al., Medium Scale Anisotropy in the TeV Cosmic Ray Flux Observed by ARGO-YBJ, Phys. Rev. D 88 (2013) 082001.

[9] HAWC Collaboration, A.U. Abeysekara et al., Observation of Small-Scale Anisotropy in the Arrival Direction Distribution of TeV Cosmic Rays with HAWC, Astrophys. J. 796 (2014) 108.

[10] IceCube Collaboration, R.U. Abbasi et al., Measurement of the Anisotropy of Cosmic-Ray Arrival Directions with IceCube, Astrophys. J. 718 (2010) L194.

[11] IceCube Collaboration, R.U. Abbasi et al., Observation of Anisotropy in the Arrival Directions of Galactic Cosmic Rays at Multiple Angular Scales with IceCube, Astrophys. J. 740 (2011) 16.

[12] IceCube Collaboration, R.U. Abbasi et al., Observation of Anisotropy in the Galactic Cosmic-Ray Arrival Directions at 400 TeV with IceCube, Astrophys. J. 746 (2012) 33.

[13] IceCube Collaboration, M.G. Aartsen et al., Observation of Cosmic-Ray Anisotropy with the IceTop Air Shower Array, Astrophys. J. 765 (2013) 55.

[14] A.D. Erlykin and A.W. Wolfendale, The Anisotropy of Galactic Cosmic Rays as a Product of Stochastic Supernova Explosions, Astropart. Phys. 25 (2006) 183.

[15] P. Mertsch and S. Funk, Solution to the Cosmic Ray Anisotropy Problem, Phys. Rev. Lett. 114 (2015) 021101.

[16] G. Giacinti and G. Sigl, Local Magnetic Turbulence and TeV-PeV Cosmic Ray Anisotropies, Phys. Rev. Lett. 109 (2012) 071101.

[17] M. Ahlers, Anomalous Anisotropies of Cosmic Rays from Turbulent Magnetic Fields, Phys. Rev. Lett. 112 (2014) 021101.

[18] IceCube Collaboration, A. Achterberg et al., First Year Performance of the IceCube Neutrino Telescope, Astropart. Phys. 26 (2006) 155.

[19] IceCube Collaboration, R.U. Abbasi et al., IceTop: The Surface Component of IceCube, Nucl. Instr. Meth. A 700 (2013) 188.

[20] J.L. Kelley for the IceCube Collaboration, Event Triggering in the IceCube Data Acquisition System, AIP Conference Proceedings 1630 (2014) 154.

[21] IceCube Collaboration, R.U. Abbasi et al., Seasonal Variations of High Energy Cosmic Ray Muons Observed by the IceCube Observatory as a Probe of Kaon/Pion Ratio, Proc. 32nd ICRC, Beijing, China (2011) [arXiv:1111.2735].

[22] K.M. Górski et al., HEALPix - a Framework for High Resolution Discretization, and Fast Analysis of Data Distributed on the Sphere, Astrophys. J. 622 (2005) 759.

[23] T.P. Li and Y.Q. Ma, Analysis Methods for Results in Gamma-Ray Astronomy, Astrophys. J. 272 (1983) 317.

[24] Tibet AS $\gamma$ Collaboration, M. Amenomori et al., On Temporal Variations of the Multi-TeV Cosmic Ray Anisotropy Using the Tibet III Air Shower Array, Astrophys. J. 711 (2010) 119.

[25] IceCube Collaboration, M.G. Aartsen et al., Study of the Time-Dependence of the Cosmic-Ray Anisotropy with AMANDA and IceCube, Proc. 33rd ICRC, Rio de Janeiro, Brazil (2013) [arXiv:1309.7006]. 